\title{
Cepstral Blur Identification by Neural Network for Image Restoration Purpose
}

\section{Sławomir Skoneczny and Rafał Foltyniewicz}

Institute of Control \& Industrial Electronics, Warsaw University of Technology, 00-662 Warszawa, ul. Koszykowa 75, Poland tel. (+48-2) 6280665, fax. (+48-2) 6256633, e-mail: skonecz@plwatu21.bitnet or rfoltyn@plwatu21.bitnet

\begin{abstract}
A deterministic shift-invariant linear blur is a kind of degradation very often encountered in image processing as a serious problem we must deal with. Examples of such degradation are: camera-motion blur, out-of-focus blur and atmospheric turbulence blur. The exact knowledge of blur type is required for restoration purposes. After restoration we would like to have the perfect image, which is obviously impossible in practice. Instead of that, many sophisticated techniques must be applied in order to obtain satisfying version of the picture but still far from degraded original not available in most cases. The crucial thing is to identify blur type and its parameters. The more accurate we can describe blur phenomenon of degraded image the more precisely we can construct proper filters, which results in better restoration. We propose a method of identifying blur type and parameters by using neural networks which learns and recognizes power cepstra of sums of eigenimages obtained in Singular Value Decomposition. A comparison to learning and recognizing of power cepstra of degraded images without SVD by neural network is given. Theoretical investigations and practical results are presented.
\end{abstract}

